\title{
Double Barrel Wet Colostomy for Urinary and Fecal Diversion
}

Keywords: Double barrel wet colostomy; Urinary diversion; Fecal diversion

\begin{abstract}
A 60-year-old male who had a history of spinal cord injury received loop colostomy for fecal diversion and cystostomy for urinary diversion. Because he was diagnosed with muscle invasive bladder cancer radical cystectomy and double barrel wet colostomy was conducted. Computed tomography showed simultaneous urinary and fecal diversion and stone formation in the distal segment of colon conduit with urinary diversion.
\end{abstract}

\section{Introduction}

In patients with an advanced primary or recurrent carcinoma, double-barreled wet colostomy can be used for pelvic exenteration and urinary tract reconstruction. It is a technique that separate urinary and fecal diversion with a single abdominal stoma. Distal end of the colon was sectioned and closed and bilateral ureters were implanted into the formed colon conduit. This technique can reduce ascending infection by avoiding fecal reflux to pelvis.

\section{Clinical Image}

A 60-year-old male presented to the emergency department with bloody urine in urinary bag for one day. He had a history of spinal cord injury due to traffic accident with paraplegia for over 30 years. He received loop colostomy for fecal diversion and cystostomy for urinary diversion. Computed Tomography (CT) showed loop colostomy with both the proximal limb and the distal limb open into the common stoma opening (Figure 1A). Fibero cystoscopy showed irregular papillary tumors over posterior wall (Figure 1B). He was diagnosed with muscle invasive bladder cancer by transurethral resection of bladder tumour and radical cystectomy with pelvis lymph node dissection was done. Pathology report showed urothelial carcinoma with squamous differentiation, high grade, TNM stage: pT2a N0 stage II. Additionally double barrel wet colostomy was conducted. Distal end of the colon was sectioned and closed at about $13 \mathrm{~cm}$ distal to the stoma and bilateral ureters were implanted into the formed colon conduit. CT after six months showed simultaneous urinary and fecal diversion and stone formation in the distal segment of colon conduit with urinary diversion (Figure 1C). The formation of calculi might be due to urinary stasis after urinary surgical diversion.

\section{Journal of}

Urology \& Nephrology

\section{Yu-Hao Xue and Chih-Hsiung Kang*}

Department of Urology, Chang Gung Memorial Hospital-Kaohsiung Medical Center, Chang Gung University College of Medicine, Kaohsiung, Taiwan, Republic of China

Address for Correspondence

Chih-Hsiung Kang, Department of Urology, Chang Gung Memorial Hospital - Kaohsiung Medical Center, Chang Gung University College of Medicine, Taiwan, E- mail: chkang5801@gmail.com

Submission: 30 October, 2017

Accepted: 06 November, 2017

Published: 10 November, 2017

Copyright: (๑) $2017 \mathrm{Kang} \mathrm{CH}$, et al. This is an open access article distributed under the Creative Commons Attribution License, which permits unrestricted use, distribution, and reproduction in any medium, provided the original work is properly cited.

Bilateral hydroureters and mild hydronephrosis were noted and we suspected the calculi impacted in bilateral ureteto-colostomy anastomosis sites. After lithotripsy of the stones in colon conduit by cystoscopy, hydronephrosis improved and the patient continued be monitored with smooth condition.
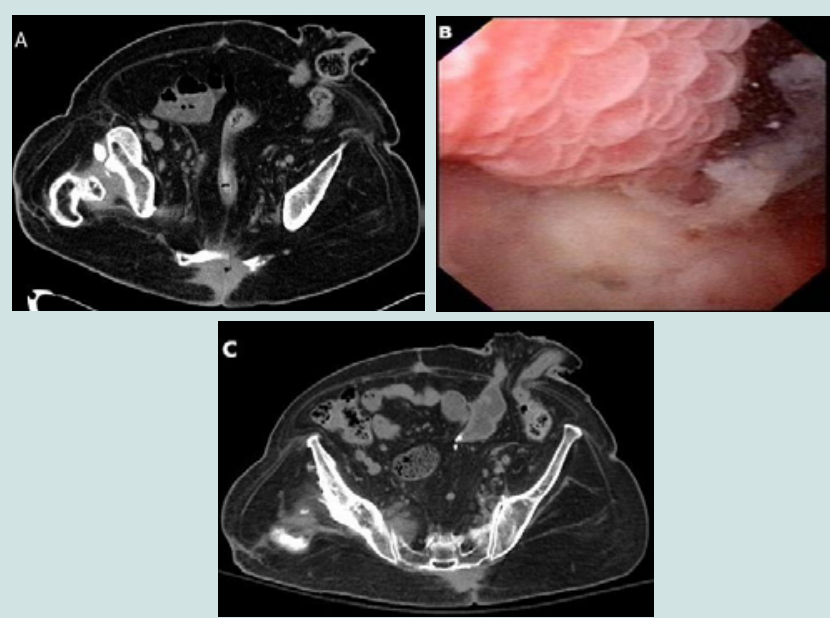

Figure 1: A: CT of the abdomen showed two segments on the loop colostomy site. B: Papillary tumors over posterior bladder wall could be seen by fibero cystoscopy. C: Post double-barreled wet colostomy six months, CT showed urinary and fecal diversion and urinary stone in the distal segment of colon conduit. 